\title{
Behavioral sequelae to early postnatal cytomegalovirus infection in mice
}

\author{
DENNIS J. McFARLAND and JOHN HOTCHIN \\ New York State Department of Health, Albany, New York
}

\begin{abstract}
The effects of intracerebral cytomegalovirus infection on the subsequent development of spontaneous alternation and motor activity were evaluated in mice innoculated at either $\mathbf{b}$ or 12 days of postnatal age. Mice infected at both ages showed a transient depression in body weight at 26 days of age. While spontaneous alternation initially developed in a normal manner, animals of both sexes infected at 5 but not 12 days of age showed a subsequent decline in this measure at 46 days of age. In contrast to the dependence of alternation effects on age at infection, hyperactivity was found to be sex dependent. These results provide a further example of the interaction between organismic factors and the behavioral effects of CNS viral infection. In addition, certain of the effects of murine cytomegalovirus infection are of a delayed nature.
\end{abstract}

Cytomegaloviruses represent a subgroup of the herpesviruses that can be characterized by a marked degree of species specificity (Plummer, 1973). Thus, individual members of this group generally replicate only in their normal host species or in cultured cells derived from the normal host species. Cytomegalovirus is the most common cause of human intrauterine virus infection with an incidence of between $0.5 \%$ and $2.5 \%$ (Whitley \& Alford, 1979). Another 3\% to $5 \%$ of the population may be infected at or near the time of birth (Reynolds, Stagno, Hosty, Tiller, \& Alford, 1973). The presence of circulating antibody increases with age so that over $50 \%$ of individuals over 30 years of age have evidence of prior infection (Plummer, 1973).

Estimates of the incidence of adverse sequelae to perinatal CMV infection vary. For example, Hansaw and Dudgeon (1978) suggest that at least $5 \%$ to $10 \%$ of infected newborns will suffer neurological damage. MacDonald and Tobin (1978) arrived at a higher estimate by including mild handicaps such as clumsiness and hyperactivity, which often appeared late. The spectrum of effects was rather large, including signs of hepatitis with or without CNS involvement, microcephaly and cerebral palsy, epilepsy, hyperactivity, and sensory deficits. A large proportion of infants with evidence of infection appeared to develop normally, however.

As is the case with human cytomegalovirus infection, there is an age-dependent decrease in the lethality and neurotropism associated with murine cytomegalovirus infection (Davis, Krawczyk, \& Hawrisak, 1979). Intracerebral innoculation of cytomegalovirus in suckling mice has been examined by Lussier

The authors' mailing address is: Center for Laboratories and Research, New York State Department of Health, Albany, New York 12201.
(1975), who suggests that this model is appropriate for the study of virus-induced malformations of the developing nervous system. Nonfatal infections lead to mineralized lesions in the cortex and hippocampus. The author states that "healing took place without apparent sequelae."

In the present study, suckling mice were infected with murine cytomegalovirus and subsequent behavioral development was evaluated. Spontaneous alternation was examined because this patterning of behavior normally shows an orderly increase during the course of postnatal development (Douglas, 1975). Of particular interest was whether or not resulting effects depend upon the age at infection, as has been observed with herpes simplex (McFarland, Sikora, \& Hotchin, 1981).

\section{METHOD}

The Smith strain of murine cytomegalovirus was obtained from the American Type Culture Collection (ATTC VR-194), and a virus pool was formed from the third intracerebral passage in 0 day Nya:Nylar mice. For each passage, the mice were innoculated intracerebrally with virus suspension, and the brains were removed 7 days later and homogenized in Hank's balanced salt solution with $0.05 \%$ gelatin to form a $10 \%$ suspension. Intracerebral injections were as standardly employed in virological investigations. These were hand-guided innoculations into the cerebral hemispheres. Examination of animals injected with various dyes indicated that this procedure produced mainly an intraventricular and subarachnoid injection. This pool contained $5 \times 10^{5}$ infectious units/milliliter as assayed in mouse fibroblast cell cultures and 3.4 $\times 10^{3} \mathrm{LD}_{\mathrm{so}} / \mathrm{ml}$ as assayed by intracerebral innoculation in 0 -day Nya:Nylar mice.

Litters of five suckling Nya:Nylar mice were innoculated at either Postnatal Day 5 or Postnatal Day 12. Separate groups of animals were innoculated intracerebrally with a $0.01-\mathrm{ml}$ volume of either a $10^{-2}$ dilution of the virus pool or a $10^{-2}$ dilution of normal suckling mouse brain. Twelve litters were innoculated at each age with cytomegalovirus, and six control litters were innoculated at each age.

Throughout the course of the study, the animals were housed in 
plastic suspended cages with their littermates. Mothers were removed from the litter on Postnatal Day 21. At all times, food and water were available ad lib and the room was maintained on a 10 h-on/14-h-off lighting schedule.

For behavioral testing, one male and one female mouse were selected randomly from each litter. Each of these animals were weighed and given a 20 -min test of $Y$-maze exploration at 18, 26, and 46 days of age (a given animal was run at each age). Exploration in photocell cages was examined in a single 20 -min session at 56 days of age. All testing was conducted during the light phase and was accomplished with the aid of an ACT/INTERACT laboratory computer system. The subjects were run in a blockrandomized design with respect to treatment and time of day.

Spontaneous alternation was examined in a symmetrical Plexiglas Y-maze, painted flat black, with $27 \times 7.5 \times 10 \mathrm{~cm}$ arms. Photocells, illuminated by infrared LEDs, were located $10 \mathrm{~cm}$ within each arm and $2.5 \mathrm{~cm}$ from the floor. The system was programmed not to count two or more successive breakings of the same photocell. The mouse was placed at the far end of one arm and the maze was covered. The total number of arms entered and the number of least recently entered arms (alternations) were recorded during a 20-min session. From this data, the percentage of alternation responses was calculated by the formula: percent alternation $=$ (alternation responses $\div$ total responses) $\times 100$.

Spontaneous motor activity was examined in $43 \times 24 \times 20 \mathrm{~cm}$ clear plastic cages trisected by two photocell units located $2 \mathrm{~cm}$ from the cage floors. These cages were painted flat black except for small areas around the photocell units, which were covered with dark red gelatin filters. The system was programmed to count only successive interruptions of the two photocell units so as to reduce sensitivity to such behaviors as sniffing and grooming in the path of the light beam. During testing, the cages were covered with a piece of white translucent Plexiglas and illuminated by overhead fluorescent lights.

\section{RESULTS}

As there were no significant differences between control animals innoculated at 5 and 12 days of age on any of the measures examined, these two groups were pooled in subsequent analyses.

The effects of cytomegalovirus infection on weight gain are shown in Table 1. An analysis of variance, with sex and treatment as between-subjects effects and age as a within-subjects effect, indicated that both the main effect of treatment $(p<.001)$ and the interaction of treatment with age $(p<.01)$ were significant. Post hoc analyses (least significant difference tests) indicated that animals infected at either 5 or 12 days of age showed significantly reduced body weights at 26 , but not 18 or 46 , days of age $(p<.01$ in both cases).

The effects of cytomegalovirus infection on the development of spontaneous alternation behavior are shown in Table 2. Analysis of variance, with sex and treatment as between-subjects effects and age as a within-subjects effect, indicated that both the main effect of age $(p<.01)$ and the treatment by age interaction $(\mathrm{p}<.01)$ were significant. Post hoc analyses indicated that animals infected at 5 days of age showed significantly reduced alternation levels at 46 days of age $(p<.05)$. Within-treatment comparison indicated that both control animals and mice infected with cytomegalovirus on Day 12 showed a significantly greater level of alternation on Day 46 than on Day 18 ( $p<.01$ in both cases). In contrast, animals infected on Day 5 showed a significant decline in alternation on Day 46 from that observed on Day 26 $(p<.05)$. An analysis of variance on the number of $\mathrm{Y}$-maze arms entered indicated that the main effect of treatment and the interactions of treatment with other variables in the analysis were not significant.

Table 1

Body Weight (in Grams) in Cytomegalovirus-Infected and Control Mice as a Function of Age (in Days)

\begin{tabular}{|c|c|c|c|c|c|c|c|}
\hline \multirow[b]{3}{*}{ Group } & \multirow[b]{3}{*}{ Sex } & \multicolumn{6}{|c|}{ Age } \\
\hline & & \multicolumn{2}{|c|}{18} & \multicolumn{2}{|c|}{26} & \multicolumn{2}{|c|}{46} \\
\hline & & Mean & SE & Mean & $\mathrm{SE}$ & Mean & $\mathrm{SE}$ \\
\hline Control & Male & 7.65 & 0.27 & 12.79 & 0.55 & 21.43 & 0.53 \\
\hline Control & Female & 7.28 & 0.21 & 11.96 & 0.26 & 19.34 & 0.49 \\
\hline 5-Day Infected & Male & 6.52 & 0.23 & 10.08 & 0.70 & 20.20 & 0.47 \\
\hline 5-Day Infected & Female & 6.94 & 0.28 & 10.07 & 0.43 & 18.58 & 0.38 \\
\hline 12-Day Infected & Male & 7.22 & 0.28 & 11.55 & 0.58 & 20.71 & 0.38 \\
\hline 12-Day Infected & Female & 7.07 & 0.21 & 10.55 & 0.45 & 18.67 & 0.52 \\
\hline
\end{tabular}

Table 2

Spontaneous Alternation in Cytomegalovirus-Infected and Control Mice as a Function of Age (in Days)

\begin{tabular}{|c|c|c|c|c|c|c|c|}
\hline \multirow[b]{3}{*}{ Group } & \multirow[b]{3}{*}{$\operatorname{Sex}$} & \multicolumn{6}{|c|}{ Age } \\
\hline & & \multicolumn{2}{|c|}{18} & \multicolumn{2}{|c|}{26} & \multicolumn{2}{|c|}{46} \\
\hline & & Mean & SE & Mean & SE & Mean & $\mathrm{SE}$ \\
\hline Control & Male & 61.1 & 3.35 & 61.7 & 4.25 & 73.3 & 2.81 \\
\hline Control & Female & $\$ 4.2$ & 5.28 & 68.6 & 3.89 & 70.0 & 3.01 \\
\hline 5-Day Infected & Male & 61.8 & 5.69 & 61.0 & 3.77 & 54.6 & 4.31 \\
\hline 5-Day Infected & Female & 59.4 & 3.19 & 68.1 & 3.51 & 58.7 & 4.56 \\
\hline 12-Day Infected & Male & 62.4 & 3.02 & 63.5 & 4.53 & 65.3 & 2.77 \\
\hline 12-Day Infected & Female & 50.4 & 5.31 & 66.8 & 4.16 & 68.6 & 2.73 \\
\hline
\end{tabular}


Table 3

Activity (Photocell Interruptions) in Cy tomegalovirus-Infected and Control Mice at 56 Days of Age

\begin{tabular}{lrrrrr}
\hline & \multicolumn{2}{c}{ Males } & & \multicolumn{2}{c}{ Females } \\
\cline { 2 - 3 } \cline { 5 - 6 } \multicolumn{1}{c}{ Group } & Mean & SE & & Mean & SE \\
\hline Control & 103.3 & 11.1 & & 96.3 & 6.38 \\
5-Day Infected & 90.2 & 5.07 & & 127.8 & 11.57 \\
12-Day Infected & 94.1 & 4.76 & & 123.5 & 13.91 \\
\hline
\end{tabular}

The effects of cytomegalovirus infection on spontaneous activity, as measured in the photocell cages, is shown in Table 3. Analysis of variance with sex and treatment as between-subjects effects indicated that both the main effects of sex $(p<.05)$ and the treatment $\times$ sex interaction $(p<.05)$ were significant. Post hoc analyses indicated that female mice infected at either 5 or 12 days of age were significantly more active than controls $(p<.05)$, whereas no significant differences were observed between male mice infected at either age.

\section{DISCUSSION}

Mice infected with cytomegalovirus at either 5 or 12 days of age showed a transient depression in body weight at 26 days of age. In contrast, decreased spontaneous alternation was not observed until $\mathbf{4 6}$ days of age and occurred only in mice infected at 5 days of age. Thus, the effects on body weight can be dissociated both in time and in terms of the dependence of the alternation effect on the age at infection. In addition, it is of interest that the alternation effect appeared following the initial normal development of this behavior in 5-day infected animals. Such an effect is thus appropriately described as a developmental regression rather than as a developmental delay. Finally, the effects of cytomegalovirus infection on activity as measured in photocell cages was found to depend upon the sex of the animal rather than on the age at infection. Since activity differences were not observed in the $\mathrm{Y}$-maze, these results could be due either to a task-dependent effect or to the fact that photocell cage activity was examined at a later age.

A consideration of the $Y$-maze data suggests that age at infection is a critical determinant of behavioral outcome, while the photocell cage data suggests that the activity effects depend upon the sex of the host. Comparing both effects, it is obvious that neither age at infection nor sex determine the outcome of cytomegalovirus infection in a unitary fashion. That is, these organismic variables do not simply influence whether or not behavioral effects occur. These results thus point to the complex interaction between the organismic factor and the behavioral response to infection, as has been previously observed with other forms of brain damage (e.g., Donovick \& Burright, 1982).

Since the behavioral development of the organism may initially proceed in a relatively normal fashion following perinatal infection, it would appear to be necessary to consider late-developing effects in attempting to evaluate adverse sequelae. As noted earlier, MacDonald and Tobin (1978) have reported that certain handicaps in cytomegalovirus-infected infants, such as "clumsiness," appear later. Such effects could conceivably be due to a susceptibility to both a benign early infection and some later, but not causally related, event. However, the present results, obtained with animals, support a concept of latedeveloping effects of perinatal infection.

\section{REFERENCES}

Davis, G., Krawczyk, K., \& Hawrisak, M. (1979). Age-related neurotropism of mouse cytomegalovirus in explanted trigeminal ganglions. American Journal of Pathology, 97, 261-272.

Donovick, P. J., \& Burhight, R. G. (1982). Genetic influences in responses to brain lesions. In I. Lieblich (Ed.), The genetics of the brain. Amsterdam: Elsevier.

Douglas, R. J. (1975). The development of hippocampal function: Implications for theory and therapy. In R. L. Isaacson \& K. H. Pribram (Eds.), The hippocampus (Vol. 2, pp. 327-362). New York: Plenum Press.

Hansaw, J., \& Dudgeon, J. (1978). Viral diseases of the fetus and newborn. Philadelphia: Saunders.

Lussier, G. (1975). Animal model: Murine cytomegalovirusinduced cerebral calcification. American Journal of Pathology, 80, 555-558.

MacDonald, H., \& Tobin, J. O. (1978). Congenital cytomegalovirus infection: A collaborative study on epidemiological, clinical and laboratory findings. Developmental Medicine and Child Neurology, 20, $471-482$.

McFarland, D. J., Sikora, E., \& Hotchin, J. (1981). Age of infection as a determinant of the behavioral effects of herpes encephalitis in mice. Physiological Psychology, 9, 87-89.

Plummer, G. (1973). Cytomegalovirus of man and animals. Progress in Medical Virology, 15, 92-125.

Reynolds, D., Stagno, S., Hosty, T. S., Tiller, M., \& Alfond, C. A. (1973). Maternal cytomegalovirus excretion and perinatal infection. New England Journal of Medicine, 289, 1-5.

Whitley, R. J., \& Alford, C. A. (1979). Chronic intrauterine and perinatal infections. In G. J. Galasso (Ed.), Antiviral agents and viral diseases of man. New York: Raven Press.

(Manuscript received August 15, 1983; revision accepted for publication December 21,1983 ). 